\title{
Embolización de la arteria renal: Serie de casos y revisión de la literatura
}

\author{
Víctor Matus B. ${ }^{1}$, Matías Kirmayr Z. ${ }^{2}$, Cecilia Retamal N. ${ }^{2}$, Laura Vergara-Merino ${ }^{2,3}$, \\ Alejandro Kotlik A. ${ }^{1}$ y Javier Pérez-Bracchiglione ${ }^{3}$
}

\section{Renal artery embolization: Case series and literature review}

Introduction: Renal artery embolization (RAE) is a percutaneous procedure that occludes the renal artery, with consequent ischemia of the vascular territory. The most common indications include hematuria and palliation for metastatic renal cancer. Despite technical development and progressive experience, studies include a small number of patients and few cases have been published in our country. Aim: To share our experience at Valparaíso-San Antonio Health Service and to review the existing literature. Materials and Method: We performed a retrospective descriptive review of medical records of patients with severe anemia due to hematuria managed with RAE, between 2012 and 2020. Subsequently, we conducted a literature search in PubMed, from inception until April 2020. Results: We included 9 patients. There were $6(66.7 \%)$ males and $3(33.3 \%)$ females with a median age of 69 years (IQR $=18)$. Main cause of hematuria was advanced kidney cancer ( 7 patients). There were no complications and clinical success was achieved in all patients. Our literature search yielded 571 references, 24 met our eligibility criteria. The age of patients and the underlying causes of hematuria were varied. The lowest clinical success rate was $65 \%$, however, 15 studies $(62.5 \%)$ reported a success equal to or greater than $90 \%$. Six studies reported more than $10 \%$ of patients with complications. Conclusion: Our results and the studies reviewed show that RAE appears to be safe and effective in the management of patients with severe anemia due to hematuria.

Keywords: embolization; therapeutic; renal artery; hematuria; review.

\section{Resumen}

Introducción: La embolización de arteria renal (EAR) es un procedimiento percutáneo que ocluye la arteria renal, con la consecuente isquemia del territorio vascular. Sus indicaciones más comunes son la hematuria y el manejo paliativo en cáncer renal metastásico. A pesar del desarrollo técnico y de la experiencia progresiva, los estudios incluyen un número reducido de pacientes y en nuestro país se revisan casos aislados. Objetivo: Describir la experiencia en el Servicio de Salud Valparaíso San Antonio y revisar la literatura existente. Materiales y Método: Realizamos un estudio descriptivo de los pacientes sometidos a EAR por anemia severa secundaria a hematuria, durante los años 2012 a 2020 . Posteriormente, realizamos una revisión de la literatura en PubMed, hasta abril de 2020. Resultados: Incluimos 9 pacientes, $6(66,7 \%)$ hombres y $3(33,3 \%)$ mujeres. La mediana de edad fue de 69 años (RIC = 18). La principal causa de la hematuria fue cáncer renal avanzado ( 7 pacientes). No hubo complicaciones, y se logró éxito clínico en todos los pacientes. Nuestra búsqueda de literatura arrojó 571 referencias y 24 cumplieron con nuestros criterios de elegibilidad. La edad de los pacientes y las causas subyacentes de hematuria fueron variadas. La menor tasa de éxito clínico fue de $65 \%$, sin embargo, 15 estudios $(62,5 \%)$ reportaron un éxito igual o mayor al $90 \%$. Seis estudios reportaron más de un $10 \%$ de pacientes con alguna complicación. Conclusión: Nuestros resultados y la evidencia revisada muestran que la EAR parece ser segura y eficaz en el manejo de anemia severa secundaria a hematuria.

Palabras clave: embolización; terapéutica; arteria renal; hematuria; revisión.
Servicio de Cirugía Hospital Eduardo Pereira. ${ }^{2}$ Interno (a) Escuela de Medicina Universidad de Valparaíso.

${ }^{3}$ Centro Interdisciplinario de Estudios en Salud (CIESAL) Universidad de Valparaíso. Valparaíso, Chile.

Recibido 2020-11-27 aceptado 2021-06-03

Correspondencia a: Dr. Javier Pérez-Bracchiglione javier.perez@uv.cl 


\section{Introducción}

La embolización de arteria renal corresponde a un procedimiento terapéutico en el que por vía percutánea y bajo visión radiológica se accede a los vasos definidos y se administran sustancias que llevan a la oclusión del lumen, con la consecuente isquemia o infarto agudo del territorio vascular ${ }^{1}$. Existen dos tipos principales de embolización: 1) Total, que ocupa el lumen de la arteria renal principal distal al ostium y 2) Parcial, que puede ser selectiva cuando es a nivel segmentario o supraselectiva cuando es a nivel de vasos subsegmentarios ${ }^{2}$.

Desde la descripción de la embolización percutánea de arterias renales en animales en 1969 por Lalli et al. ${ }^{3}$, y la primera descrita en humanos en 1973 por Almgard et al. ${ }^{4,5}$, se han descrito múltiples indicaciones, siendo la hematuria y el manejo paliativo en cáncer renal metastásico las más clásicas. Sin embargo, existen otras indicaciones: terapia adyuvante en cáncer renal (citorreducción), contraindicación anestésica o rechazo por el paciente de nefrectomía quirúrgica, tratamiento de angiomiolipomas, malformaciones arteriovenosas, fístulas arteriovenosas, enfermedad renal médica, trauma, complicaciones postrasplante, entre otras ${ }^{1,2,6}$. La Sociedad Europea de Urología no incluye actualmente recomendaciones para embolización de arteria renal por hematuria ${ }^{7}$.

A pesar del desarrollo técnico y de la experiencia intervencional progresiva, los estudios internacionales incluyen un número reducido de pacientes y en nuestro país se revisan casos aislados de embolizaciones de arterias renales.

El objetivo del presente artículo es describir la experiencia local, caracterizando a la población que ha sido sometida a embolización de arterias renales en el Servicio de Salud Valparaíso-San Antonio y revisar la literatura existente.

\section{Materiales y Método}

Realizamos un estudio descriptivo de los pacientes con indicación terapéutica de embolización percutánea de arteria renal por anemia severa secundaria a hematuria y fracaso a terapia médica, pertenecientes al Hospital Carlos Van Buren y al Hospital Eduardo Pereira de Valparaíso, durante los años 2012 a 2020. En relación con procedimiento realizado, los pacientes estuvieron hospitalizados por al menos $24 \mathrm{~h}$, en todos se realizó el procedimiento en pabellón de Arco $\mathrm{C}$ bajo sedación y anestesia local, el acceso femoral fue por punción no ecoguiada con introductor 6Frx55. Los catéteres utilizados fueron Pigtail, Cobra hidrofílico, Kumpe o Vertebral, de acuerdo a la anatomía. Se utilizó como material embolizante coils (en aquellos casos en que no se informa el material fue por falta de registro de éste). Posteriormente se realizó hemostasia por compresión en la mayoría de los casos como se menciona en la sección de resultados, sin ser necesario el uso de suturas percutáneas. Describimos las siguientes variables: Sexo, edad, lateralidad del procedimiento, motivo del procedimiento, necesidad de arteriotomía, complicaciones durante el período intrahospitalario, días de estadía hospitalaria y éxito clínico del procedimiento. Describimos todas las variables con número absoluto y porcentajes, y mediana y rangos intercuartiles (RIC) según corresponda.

Posteriormente, realizamos una revisión de la literatura en PubMed, desde el origen hasta abril de 2020, buscando estudios primarios experimentales, observacionales o descriptivos que incluyan $10 \mathrm{pa}-$ cientes o más y caractericen al menos el sexo, edad, causa de la hematuria, éxito clínico del procedimiento y complicaciones de los pacientes sometidos a embolización de arteria renal por anemia secundaria a hematuria severa. La estrategia de búsqueda se presenta en el Anexo 1.

\section{Resultados}

Incluimos nueve pacientes durante el período de estudio. Seis pacientes $(66,7 \%)$ eran hombres, y tres $(33,3 \%)$ mujeres. La mediana de edad fue de 69 años $(\mathrm{RIC}=18)$. En cuatro pacientes $(44,4 \%) \mathrm{se}$ realizó el procedimiento en la arteria renal izquierda, y en cinco pacientes $(55,6 \%)$ en la derecha. En ningún paciente se realizó el procedimiento en forma bilateral. La principal causa de la hematuria fue cáncer renal avanzado en siete pacientes $(77,8 \%)$, hipervascularización en un paciente $(11,1 \%)$, y fístula arteriovenosa por nefrostomía percutánea en un paciente $(11,1 \%)$. Se utilizó una mediana de 63 mililitros $(\mathrm{RIC}=20)$ de medio de contraste en los pacientes sometidos al procedimiento.

De los siete pacientes que tenían registrado uso de coils, en seis $(85,7 \%)$ se utilizaron cuatro coils y en uno $(14,3 \%)$ se utilizaron seis. En cuanto al método de hemostasia del procedimiento, en tres pacientes $(33,3 \%)$ se registró compresión por $15 \mathrm{~min}$, en un paciente $(11,1 \%)$ se realizó gareta a arteria femoral, y en cinco $(55,6 \%)$ no se registró método de hemostasia. Todos tuvieron un día de estadía hospitalaria, ningún paciente tuvo complicaciones 
previas al alta hospitalaria, y se logró éxito clínico en los nueve pacientes.

Nuestra búsqueda de literatura arrojó un total de 571 referencias. Tras un cribado en base a título y resumen, excluimos 278 por tener una población o intervención distinta, 183 por ser reportes de un caso y 50 por reportar menos de 10 casos de emboliza- ción de arteria renal por hematuria. Revisamos el texto completo de 54 de las 60 referencias restantes, ya que no pudimos acceder a seis, explicamos los motivos de exclusión de cada una en el Anexo 2. Finalmente, 24 referencias cumplieron con nuestros criterios de elegibilidad $^{8-30}$.

La Tabla 1 muestra las principales características

Tabla 1. Características generales de los estudios

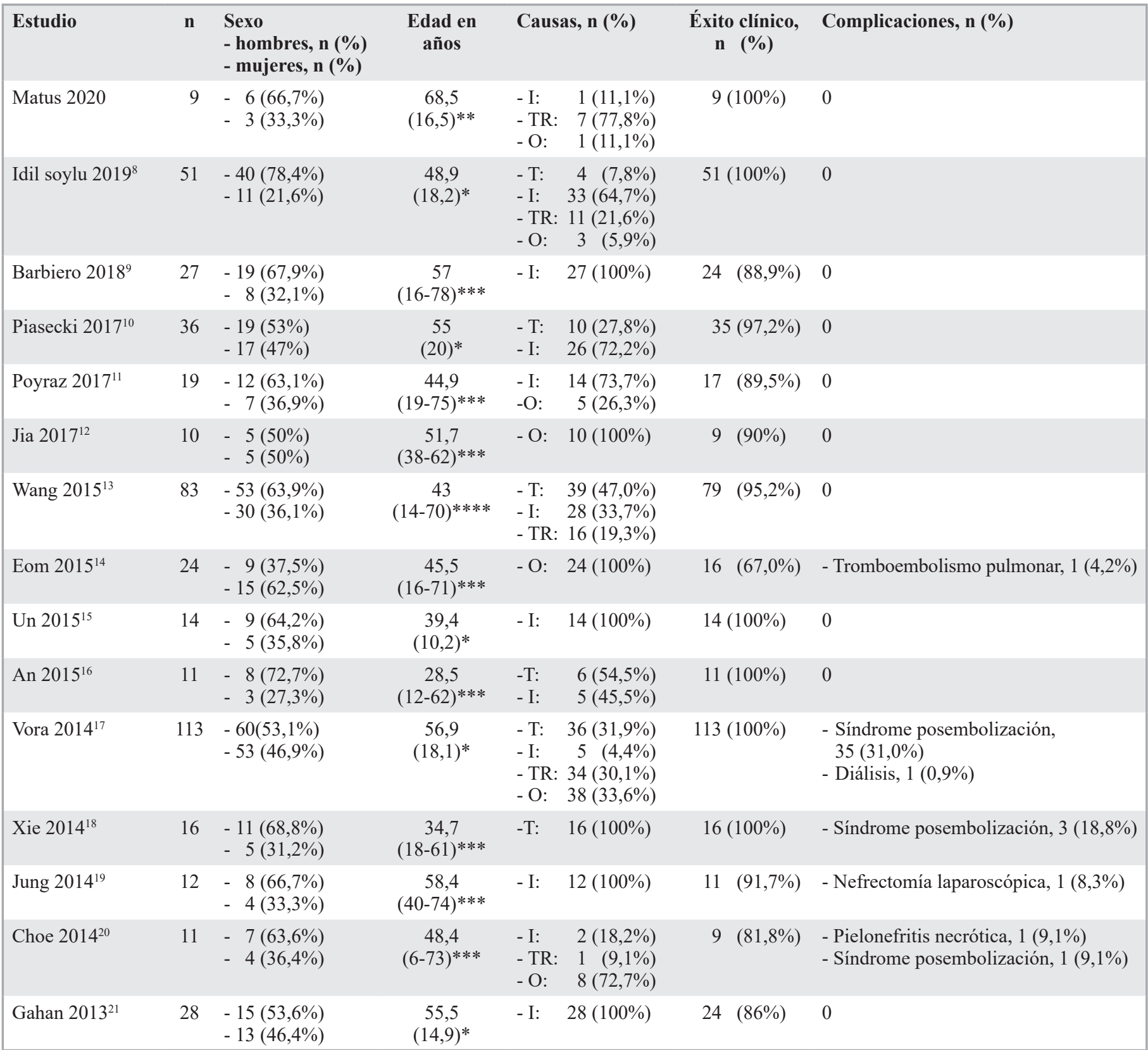




\section{SERIE DE CASOS}

\begin{tabular}{|c|c|c|c|c|c|c|}
\hline Estudio & $\mathbf{n}$ & $\begin{array}{l}\text { Sexo } \\
\text { - hombres, n (\%) } \\
\text { - mujeres, n (\%) }\end{array}$ & $\begin{array}{l}\text { Edad en } \\
\text { años }\end{array}$ & Causas, n (\%) & $\begin{array}{l}\text { Éxito clínico, } \\
\text { n }(\%)\end{array}$ & Complicaciones, n (\%) \\
\hline Jinga $2013^{22}$ & 22 & $\begin{array}{l}-15(68,2 \%) \\
-\quad 7(31,8 \%)\end{array}$ & $\begin{array}{c}52 \\
(28-69) * * *\end{array}$ & - I: $\quad 22(100 \%)$ & $21 \quad(95,5 \%)$ & - Nefrectomía de emergencia, 1 (4,5\%) \\
\hline Huang $2013^{23}$ & 12 & $\begin{array}{l}-7(58,3 \%) \\
-\quad 5(41,7 \%)\end{array}$ & $\begin{array}{c}45 \\
(28-65)^{* * *}\end{array}$ & $12(100 \%)$ & $12(100 \%)$ & 0 \\
\hline Sayani $2012^{25}$ & 32 & $\begin{array}{l}-19(59,4 \%) \\
-13(31,6 \%)\end{array}$ & $\begin{array}{c}48,2 \\
(15,4)^{*}\end{array}$ & $\begin{array}{lr}\text { - T: } & 2(6,3 \%) \\
\text { - I: } & 16(50 \%) \\
\text { - TR: } & 4(12,5 \%) \\
\text { - O: } & 10(31,2 \%)\end{array}$ & $30(93,8 \%)$ & - Desplazamiento coil, $1(3,1 \%)$ \\
\hline Liu $2009^{26}$ & 16 & $\begin{array}{l}-11(68,7 \%) \\
-5(31,3 \%)\end{array}$ & $\begin{array}{c}43,8 \\
(32-69) * * *\end{array}$ & $\begin{array}{lrr}\text { - T: } & 1 & (6,25 \%) \\
\text { - I: } & 11 & (68,75 \%) \\
\text { - TR: } & 1 & (6,25 \%)\end{array}$ & $16(100 \%)$ & - Insuficiencia renal, $3(18,8 \%)$ \\
\hline Mohsen $2007^{28}$ & 81 & $\begin{array}{l}-66(81,5 \%) \\
-15(18,5 \%)\end{array}$ & $\begin{array}{c}38 \\
(10-76) * * *\end{array}$ & $\begin{array}{ll}\text { - T: } & 19(23,5 \%) \\
\text { - I: } & 62(76,5 \%)\end{array}$ & 71 (88\%). & $\begin{array}{l}\text { - Hematoma perinefrítico, } 4(4,9 \%) \\
\text { - Hemorragia no controlada, } 2(2,5 \%)\end{array}$ \\
\hline Takebayashi $1998^{29}$ & 30 & $\begin{array}{l}-6(20 \%) \\
-24(80 \%)\end{array}$ & $\begin{array}{c}40 \\
(24-60) * * *\end{array}$ & - O: $30(100 \%)$ & $21(70 \%)$ & $\begin{array}{l}\text { - Disnea y cefalea, } 1(3,3 \%) \\
\text { - Riñón no funcional, } 1(3,3 \%) \\
\text { - Tromboembolismo pulmonar, } 1(3,3 \%)\end{array}$ \\
\hline Vorwerk $1993^{30}$ & 22 & $\begin{array}{l}-15(68,1 \%) \\
-\quad 7(31,9 \%)\end{array}$ & $\begin{array}{c}47,5 \\
(5-73)^{* * *}\end{array}$ & $\begin{array}{lrr}\text { - T: } & 1 & (4,5 \%) \\
\text { - I: } & 20(90,9 \%) \\
\text { - TR: } & 1 \quad(4,5 \%)\end{array}$ & $21 \quad(95,4 \%)$ & $\begin{array}{l}\text { - Trombosis arterias renales no } \\
\text { afectadas, } 1(4,5 \%) \\
\text { - Espasmo vascular transitorio, } 1(4,5 \%) \\
\text { - Disección arterial, } 1(4,5 \%)\end{array}$ \\
\hline
\end{tabular}

Para describir la edad se utilizaron las medidas reportadas por los autores: *promedio y desviación estándar; **mediana y RIC; ***promedio y rango; ****mediana y rango. T: Trauma; I: Iatrogenia; TR: Tumor renal; O: Otros.

de nuestro estudio y de los estudios incluidos. Destaca que sólo uno reporta más de $100 \operatorname{casos}^{17}$, y 15 $(62,5 \%)$ reportan menos de 30 casos. En 21 estudios hubo mayor proporción de hombres que mujeres, en dos hubo una mayor proporción de mujeres y en uno la proporción fue la misma. La edad de los pacientes y las causas subyacentes de hematuria fueron muy variadas entre los estudios. En cuanto al éxito clínico del procedimiento, la menor tasa reportada es de $65 \%^{27}$, sin embargo, 15 estudios $(62,5 \%)$ reportan un éxito igual o mayor al $90 \%$ en el primer intento de embolización, entre ellos el estudio que reporta más de 100 casos. Finalmente, seis estudios reportaron más de un $10 \%$ de pacientes con alguna complicación.

\section{Discusión}

En el Servicio de Cirugía del Hospital Eduardo Pereira, se realizaron embolizaciones de arteria renal a 9 pacientes provenientes del Servicio de Urología del Hospital Carlos Van Buren por anemia secundaria a hematuria entre los años 2012 y 2020. La principal causa de hematuria en estos pacientes fue cáncer renal avanzado. El procedimiento fue exitoso en todos los pacientes, sin complicaciones asociadas.

Los casos que requieren embolización de arteria renal en nuestro país y en el mundo son pocos, lo que explica el número de pacientes observados en nuestros centros. Dado lo poco frecuente de las complicaciones del procedimiento mínimamente invasivo, este se prefiere por sobre la cirugía para el control de anemia severa asociada a hematuria, en la mayoría de los casos $^{31}$.

Destacamos que al igual que en la mayoría de los estudios revisados, hubo más hombres intervenidos que mujeres. Por otro lado, los pacientes incluidos en nuestro estudio tenían una edad mayor que los pacientes de los otros reportes, y aun así presentamos una tasa de éxito del $100 \%$, sin complicaciones 
asociadas. En cuanto a las causas de hematuria comunicadas, ningún estudio además del nuestro reportó a los tumores renales como principal causa. Esto podría deberse a una menor proporción de trauma y iatrogenia en nuestro medio o a que la población analizada en cada trabajo era distinta. Una revisión sistemática de 2017 menciona que las neoplasias renales son la causa más frecuente de hemorragia renal espontánea, y las posiciona como una importante causa de hematuria, esta es la principal etiología en nuestro medio ${ }^{31}$.

Otros estudios que incluían pacientes con otras indicaciones, además de hematuria, de embolización de arteria renal, también mostraron su efectividad y su baja tasa de complicaciones ${ }^{1,4,32-36}$. Por otro lado, los resultados de los análisis retrospectivos son a veces inconsistentes y no hay ensayos prospectivos aleatorizados que comparen los parámetros quirúrgicos entre los subgrupos que se embolizan y los que no ${ }^{1}$.

Algunas debilidades de nuestro estudio fueron el bajo número de pacientes incluidos y su carácter retrospectivo. Sin embargo, logramos abarcar ocho años de experiencia local en embolización de arterias renales por anemia severa debido a hematuria, y analizar las tasas de éxito y complicaciones en nuestros centros, siendo los primeros en exponer una serie de casos a nivel nacional. Además, realizamos una búsqueda y revisión estructurada de la literatura existente con la que pudimos complementar y comparar nuestros resultados, destacando la alta tasa de éxito clínico y baja tasa de complicaciones asociadas a la embolización de arteria renal.

Nuestro estudio permite exponer los resultados locales y mundiales de la embolización de arteria renal en pacientes con anemia severa por hematuria, y refuerza la indicación de un manejo mínimamente invasivo al enfrentarse a estos pacientes. Si bien no encontramos análisis comparativos entre la embolización de arterias renales y cirugía, los resultados expuestos orientan a una alta eficacia y seguridad del procedimiento. Existen, además, muchas otras indicaciones de embolización de arteria renal que no revisamos en este artículo, por lo que sugerimos que se siga comunicando la experiencia local y nacional al respecto para situarnos en el contexto internacional ${ }^{37}$.

\section{Conclusiones}

Con los resultados obtenidos en los Hospitales Carlos Van Buren y Eduardo Pereira, más la evidencia de estudios no comparativos retrospectivos, podemos concluir que la embolización de arterias renales parece ser segura y eficaz en el manejo de anemia severa secundaria a hematuria. Sin embargo, estudios de carácter prospectivo son necesarios para evaluar el efecto real de esta intervención.

\section{Responsabilidades éticas}

Protección de personas y animales. Los autores declaran que para esta investigación no se han realizado experimentos en seres humanos ni en animales.

Confidencialidad de los datos. Los autores declaran que en este artículo no aparecen datos de pacientes.

Conflictos de interés: no hay.

Anexo 1. Estrategia de búsqueda en PubMed

\begin{tabular}{|c|l|}
\hline$\# 1$ & $($ renal*[Title/Abstract] OR kidney*[Title/Abstract]) OR "Renal Artery”[Mesh] \\
\hline$\# 2$ & $\begin{array}{l}\text { (embolotherap*[Title/Abstract] OR embolization*[Title/Abstract] OR embolisation*[Title/Abstract]) OR } \\
\text { "Embolization, Therapeutic"[Mesh] }\end{array}$ \\
\hline$\# 3$ & (anemia*[Title/Abstract] OR hematuria[Title/Abstract]) OR (“Anemia”[Mesh] OR "Hematuria”[Mesh]) \\
\hline$\# 4$ & $\# 1$ AND \#2 AND \#3 \\
\hline
\end{tabular}




\section{SERIE DE CASOS}

Anexo 2. Referencias excluidas tras lectura texto completo

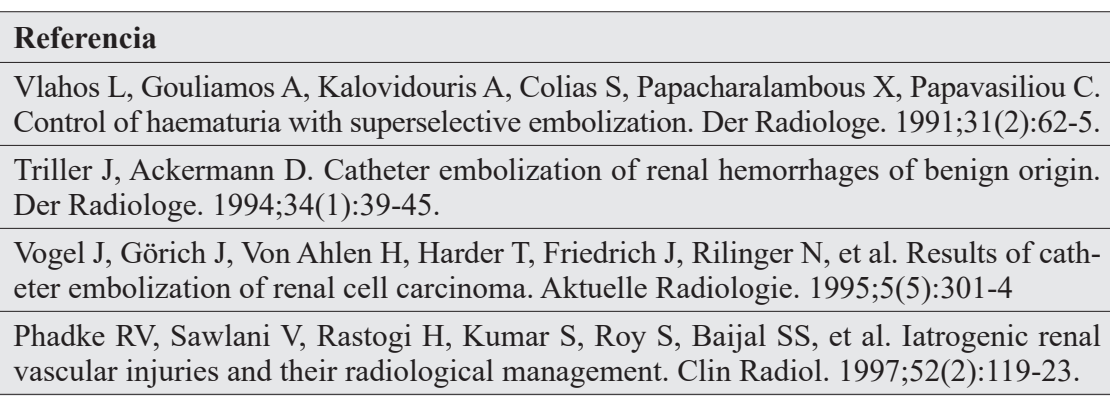

Lovaria A, Nicolini A, Meregaglia D, Saccheri S, Rivolta R, Rampoldi A, et al., editors. Interventional radiology in the treatment of urological vascular complications. Annales d'urologie; 1999.

Li Z, Yan Y, Liu T, Kong C, He X. Application of super-selective renal artery embolization in renal injuries. Chinese journal of traumatology $=$ Zhonghua chuang shang $\mathrm{za}$ zhi. 2000;3(3):166-8.

Ubara Y, Tagami T, Sawa N, Katori H, Yokota M, Takemoto F, et al. Renal contraction therapy for enlarged polycystic kidneys by transcatheter arterial embolization in hemodialysis patients. Am J Kidney Dis. 2002;39(3):571-9.

Fichtner J, Swoboda A, Hutschenreiter G, Neuerburg J. [Percutaneous embolization of the kidney: indications and clinical results]. Aktuelle Urol. 2003;34(7):475-7.

Delgado P, Diaz F, Gonzalez A, Sanchez E, Gutierrez P, Hernandez D, et al. Intolerance syndrome in failed renal allografts: incidence and efficacy of percutaneous embolization. Am J Kidney Dis. 2005;46(2):339-44.

Srivastava A, Singh KJ, Suri A, Dubey D, Kumar A, Kapoor R, et al. Vascular complications after percutaneous nephrolithotomy: are there any predictive factors? Urology. 2005;66(1):38-40.

Somani BK, Nabi G, Thorpe P, Hussey J, McClinton S. Therapeutic transarterial embolisation in the management of benign and malignant renal conditions. Surgeon. 2006;4(6):348-52.

Hallscheidt P, Besharati S, Noeldge G, Haferkamp A, Lopez R, Kauffmann GW. [Preoperative and palliative embolization of renal cell carcinomas: follow-up of 49 patients]. Rofo. 2006;178(4):391-9.

Guy L, Alfidja AT, Chabrot P, Ravel A, Boiteux JP, Boyer L. Palliative transarterial embolization of renal tumors in 20 patients. Int Urol Nephrol. 2007;39(1):47-50.

Serafin Z, Karolkiewicz M, Strzesniewski P, Lasek W, Bryczkowski M, Wolski Z. Palliative percutaneous kidney embolization with enbucrilate in patients with renal cell carcinoma: safety and symptom control. Med Sci Monit. 2007;13 Suppl 1:98-104.

Cimsit NC, Baltacioglu F, Cengic I, Akpinar IN, Ilker Y, Turkeri L. Transarterial glue embolization in iatrogenic renovascular injuries. Int Urol Nephrol. 2008;40(4):875-9.

Sakr MA, Desouki SE, Hegab SE. Direct percutaneous embolization of renal pseudoaneurysm. J Endourol. 2009;23(6):875-8.

Tillou X, Boutemy F, Remond A, Petit J. [Contribution of curative and preventive embolization for renal angiomyolipomas treatment]. Prog Urol. 2010;20(9):627-32.

Ghoneim TP, Thornton RH, Solomon SB, Adamy A, Favaretto RL, Russo P. Selective arterial embolization for pseudoaneurysms and arteriovenous fistula of renal artery branches following partial nephrectomy. J Urol. 2011;185(6):2061-5.

Tondel C, Vikse BE, Bostad L, Svarstad E. Safety and complications of percutaneous kidney biopsies in 715 children and 8573 adults in Norway 1988-2010. Clin J Am Soc Nephrol. 2012;7(10):1591-7.

Nassiri N, Dudiy Y, Carroccio A, Rosen RJ. Transarterial treatment of congenital renal arteriovenous fistulas. J Vasc Surg. 2013;58(5):1310-5.

\section{Motivo de exclusión}

Sin acceso a texto completo.

Sin acceso a texto completo.

Sin acceso a texto completo.

No describe todas las variables de interés: no describe sexo.

Sin acceso a texto completo.

Sin acceso a texto completo.

No describe pacientes con hematuria de manera independiente.

$<10$ pacientes embolizados por hematuria.

No describe pacientes con hematuria de manera independiente.

No describe pacientes embolizados de manera independiente.

No describe pacientes con hematuria de manera independiente.

$<10$ pacientes embolizados por hematuria.

No describe pacientes con hematuria de manera independiente.

No describe pacientes con hematuria de manera independiente.

No describe todas las variables de interés: no describe sexo ni edad.

No describe todas las variables de interés: no describe sexo.

$<10$ pacientes embolizados por hematuria.

No describe todas las variables de interés: no describe sexo..

No describe pacientes con hematuria de manera independiente.

No describe pacientes con hematuria de manera independiente. 
van der Wilden GM, Velmahos GC, Joseph DK, Jacobs L, Debusk MG, Adams CA, et al. Successful nonoperative management of the most severe blunt renal injuries: a multicenter study of the research consortium of New England Centers for Trauma. JAMA Surg. 2013;148(10):924-31.

Ouzaid I, Autorino R, Fatica R, Herts BR, McLennan G, Remer EM, et al. Active surveillance for renal angiomyolipoma: outcomes and factors predictive of delayed intervention. BJU Int. 2014;114(3):412-7.

Eijkemans MJ, van der Wal W, Reijnders LJ, Roes KC, van Waalwijk van Doorn-Khosrovani SB, Pelletier C, et al. Long-term Follow-up Assessing Renal Angiomyolipoma Treatment Patterns, Morbidity, and Mortality: An Observational Study in Tuberous Sclerosis Complex Patients in the Netherlands. Am J Kidney Dis. 2015;66(4):638-45.

Jaganjac S, Schefe L. Palliative embolization of renal tumors. Vojnosanit Pregl. 2015;72(12):1105-10.

Hyams ES, Pierorazio P, Proteek O, Sukumar S, Wagner AA, Mechaber JL, et al. Iatrogenic vascular lesions after minimally invasive partial nephrectomy: a multi-institutional study of clinical and renal functional outcomes. Urology. 2011;78(4):820-6.

Zhao Y, Zhong B, Xu S, Shen B, Tan F. Interventional treatment of massive hemorrhage after percutaneous nephrolithotomy. Zhonghua yi xue za zhi. 2014;94(30):2369-71.

Mao Q, Zhong B, Lin Y, Wang C, Liang W, Tan F, et al. Clinical application of computed tomographic angiography in patients with renal arterial hemorrhage: Diagnostic accuracy and subsequent therapeutic outcome. Exp Ther Med. 2015;10(2):508-12.

Zabkowski T, Piasecki P, Zielinski H, Wieczorek A, Brzozowski K, Ziecina P. Superselective renal artery embolization in the treatment of iatrogenic bleeding into the urinary tract. Med Sci Monit. 2015;21:333-7.

Vozianov S, Sabadash M, Shulyak A. Experience of renal artery embolization in patients with blunt kidney trauma. Cent European J Urol. 2015;68(4):471-7.

Gupta N, Patel A, Ensor J, Ahrar K, Ahrar J, Tam A, et al. Multiple Renal Artery Pseudoaneurysms in Patients Undergoing Renal Artery Embolization Following Partial Nephrectomy: Correlation with RENAL Nephrometry Scores. Cardiovasc Intervent Radiol. 2017;40(2):202-9.

Sun P, Liu J, Charles H, Hulbert J, Bissler J. Outcomes of angioembolization and nephrectomy for renal angiomyolipoma associated with tuberous sclerosis complex: a real-world US national study. Curr Med Res Opin. 2017;33(5):821-7.

Manoj K, Garg G, Nagathan D, Verma SK, Kohli N, Kumar S, et al. Duplex Ultrasound-guided Direct Percutaneous Management of Pseudoaneurysm of Branch of Segmental Renal Artery: Technique and Its Outcome. Urology. 2018;118:234-8.

Chavali JSS, Bertolo R, Kara O, Garisto J, Mouracade P, Nelson RJ, et al. Renal Arterial Pseudoaneurysm After Partial Nephrectomy: Literature Review and Single-Center Analysis of Predictive Factors and Renal Functional Outcomes. J Laparoendosc Adv Surg Tech A. 2019;29(1):45-50.

Kimura Y, Osuga K, Ono Y, Nakazawa T, Higashihara H, Tomiyama N. Long-Term Outcomes of Selective Renal Artery Embolization for Renal Arteriovenous Fistulae with Dilated Venous Sac. J Vasc Interv Radiol. 2018;29(7):952-7.

Chung DY, Lee JS, Ahmad A, Chang KD, Ham WS, Han WK, et al. Lessons learned from clinical outcome and tumor features of patients underwent selective artery embolization due to postoperative bleeding following 2076 partial nephrectomies: propensity scoring matched study. World J Urol. 2020;38(5):1235-42.

Saljoghi R, Le Vourch A, Renard B, Villers A, Bouye S. [Arterial embolization of polycystic kidneys as an alternative to ergonomic nephrectomy in renal pre-transplantation. Monocentric retrospective study]. Prog Urol. 2019;29(10):482-9.

Garcia-Padilla PK, Afanador D, Gonzalez CG, Yucuma D, Uribe J, Romero A, editors. Renal Graft Embolization as a Treatment for Graft Intolerance Syndrome. Transplantation Proceedings; 2020: Elsevier.
No describe pacientes embolizados de manera independiente.

No describe pacientes embolizados de manera independiente.

Población distinta: no describe si pacientes presentaban hematuria o no.

No describe pacientes embolizados de manera independiente.

No describe pacientes con hematuria de manera independiente.

No describe pacientes embolizados de manera independiente.

Sin acceso a texto completo

No describe todas las variables de interés: no describe éxito del procedimiento ni complicaciones. No describe pacientes embolizados de manera independiente.

No describe todas las variables de interés: no describe sexo ni edad.

No describe todas las variables de interés: no describe sexo ni edad.

No describe pacientes con hematuria de manera independiente.

No describe pacientes con hematuria de manera independiente.

Intervención distinta.

No describe pacientes con hematuria de manera independiente.

$<10$ pacientes embolizados por hematuria No describe pacientes con hematuria de manera independiente.

No describe pacientes con hematuria de manera independiente.

Población distinta: describe pacientes sometidos a embolización por causa distinta a hematuria.

$<10$ pacientes embolizados por hematuria. 


\section{Bibliografía}

1. Guziński M, Kurcz J, Tupikowski K, Antosz E, Słowik P, Garcarek J. The Role of Transarterial Embolization in the Treatment of Renal Tumors. Adv Clin Exp Med. 2015;24:837-43.

2. Sauk S, Zuckerman DA. Renal artery embolization. Semin Intervent Radiol. 2011;28:396-406

3. Lalli AF, Peterson N, Bookstein JJ. Roentgen-guided infarctions of kidneys and lungs. A potential therapeutic technic. Radiology 1969;93:434-5.

4. Jaganjac S, Schefe L. Palliative embolization of renal tumors. Vojnosanit Pregl. 2015;72:1105-10.

5. Almgård LE, Fernström I, Haverling M, Ljungqvist A. Treatment of renal adenocarcinoma by embolic occlusion of the renal circulation. Br J Urol. 1973;45:474-9.

6. Schwartz MJ, Smith EB, Trost DW, Vaughan ED, Jr. Renal artery embolization: clinical indications and experience from over 100 cases. BJU Int. 2007;99:881-6.

7. Ljungberg B, Cowan NC, Hanbury DC, Hora M, Kuczyk MA, Merseburger AS, et al. EAU Guidelines on Renal Cell Carcinoma: The 2010 Update. European Urology 2010;58:398-406.

8. Idil Soylu A, Uzunkaya F, Belet Ü, Akan H. Selective transarterial embolization of acute renal hemorrhage: a retrospective study. Minim Invasive Ther Allied Technol. 2020;29:326-33. doi: 10.1080/13645706.2019.1655063. Epub 2019 Aug 21.

9. Barbiero G, Groff S, Battistel M, Casarin A, Guarise A, Miotto D. Are iatrogenic renal artery pseudoaneurysms more challenging to embolize when associated with an arteriovenous fistula? Radiol Med. 2018; 123:742-52.

10. Piasecki P, Ząbkowski T, Brzozowski K, Narloch J, Zięcina P, Dziuk M, et al. The Assessment of the Risk of Acute Kidney Injury in Patients Undergoing an Urgent Endovascular Treatment Due to Severe Renal Bleeding. Cardiovasc Intervent Radiol. 2018;41:398-405.

11. Poyraz N, Balasar M, Gökmen İE, Koç O, Sönmez MG, Aydın A, et al. Clinical efficacy and safety of transcatheter embolization for vascular complications after percutaneous nephrolithotomy. Wideochir Inne Tech Maloinwazyjne. 2017;12:403-8.

12. Jia Z-Y, Zhou C-G, Xia J-G, Zhao L-B, Zhang W, Liu S, et al. Endovascular Treatment of 12 Cases of Renal Arteriovenous Malformations: The Experience of 1 Center and an Overview of the Literature. Vasc Endovascular Surg. 2018;52:46-51.

13. Wang HL, Xu CY, Wang HH, Xu W. Emergency Transcatheter Arterial Embolization for Acute Renal Hemorrhage. Medicine 2015;94:e1667.

14. Eom HJ, Shin JH, Cho YJ, Nam DH, Ko GY, Yoon HK. Transarterial embolisation of renal arteriovenous malformation: safety and efficacy in 24 patients with follow-up. Clin Radiol. 2015;70:1177-84.

15. Un S, Cakir V, Kara C, Turk H, Kose O, Balli O, et al. Risk factors for hemorrhage requiring embolization after percutaneous nephrolithotomy. Can Urol Assoc J. 2015;9(9-10):E594-8.

16. An T, Zhang S, Xu M, Zhou S, Wang W. Transcatheter embolization of peripheral renal artery for hemorrhagic urological emergencies using FuAiLe medical glue. Sci Rep. 2015;5:9106.

17. Vora A, Brodsky R, Nolan J, Ram S, Richter L, Yingling C, et al. Incidence of postembolization syndrome after complete renal angioinfarction: a single-institution experience over four years. Scand J Urol. 2014;48:245-51.

18. Rao D, Zhu H, Jin S, Yu K, Yu H, Xie L. Superselective transcatheter renal artery embolization for the treatment of hemorrhage from non-iatrogenic blunt renal trauma: report of 16 clinical cases. Therapeutics and Clinical Risk Management. Ther Clin Risk Manag. 2014;10:455-8. doi: 10.2147/ TCRM.S59671. eCollection 2014.

19. Jung S, Min GE, Chung BI, Jeon SH. Risk factors for postoperative hemorrhage after partial nephrectomy. Korean J Urol. 2014;55:17-22.

20. Choe J, Shin JH, Yoon H-K, Ko G-Y, Gwon DI, Ko HK, et al. Safety and efficacy of transarterial nephrectomy as an alternative to surgical nephrectomy. Korean J Radiol. 2014;15:472-80.

21. Gahan JC, Gaitonde M, Wadskier L, Cadeddu JA, Trimmer C. Renal function outcomes following selective angioembolization for iatrogenic vascular lesions after partial nephrectomy. J Endourol. 2013;27:1516-9.

22. Jinga $\mathrm{V}$, Dorobat $B$, Youssef $S$, Radavoi GD, Braticevici B, Filipoiu F, et al. Transarterial embolization of renal vascular lesions after percutaneous nephrolithotomy. Chirurgia 2013;108:521-

23. Huang $N$, Yang $W-Z$, Jiang N, Zheng Q-B, Huang J-Y, Shen Q. Application of Guglielmi detachable coils in embolization of iatrogenic renal hemorrhage. Ann Vasc Surg. 2013;27:1081-7

24. Murata S, Onozawa S, Nakazawa K, Akiba A, Mine T, Ueda T, et al. Endovascular embolization strategy for renal arteriovenous malformations. Acta Radiol. 2014;55:71-7.

25. Sayani R, Azeemuddin M, ul Haq T, Hamid RS, Salam B. An institutional review of transarterial embolization in haemorrhagic urological emergencies. J Pak Med Assoc. 2012;62:107-11.

26. Liu F-Y, Wang M-Q, Fan Q-S, Wang Z-J, Duan F, Song P. Emergency intervention therapy for renal vascular injury. Chin J Traumatol. 2009;12:81-6.

27. Breyer BN, McAninch JW, Elliott SP, Master VA. Minimally invasive endovascular techniques to treat acute renal hemorrhage. J Urol. 2008;179:224852; discussion 53.

28. Mohsen T, El-Assmy A, El-Diasty T. Long-term functional and morphological effects of transcatheter arterial embolization of traumatic renal vascular injury. BJU Int. 2008;101:473-7.

29. Takebayashi S, Hosaka M, Kubota Y, Ishizuka E, Iwasaki A, Matsubara S. Transarterial embolization and ablation of renal arteriovenous malformations: efficacy and damages in 30 patients with long-term followup. J Urol. 1998;159:696701.

30. Vorwerk D, Günther RW, Keulers P, Jakse G. Superselective kidney artery embolization as therapy of choice in bleeding of benign origin. Dtsch Med Wochenschr. 1993;118:325-9.

31. Ahn T, Roberts MJ, Navaratnam A, Chung E, Wood S. Changing etiology and management patterns for spontaneous renal hemorrhage: a systematic review of 
contemporary series. Int Urol Nephrol. 2017;49:1897-905.

32. Munro NP, Woodhams S, Nawrocki JD, Fletcher MS, Thomas PJ. The role of transarterial embolization in the treatment of renal cell carcinoma. BJU Int. 2003;92:240-4.

33. Gallucci M, Guaglianone S, Carpanese L, Papalia R, Simone G, Forestiere E, et al. Superselective Embolization as First Step of Laparoscopic Partial Nephrectomy. Urology 2007;69:642-5.

34. Maxwell NJ, Saleem Amer N, Rogers E, Kiely D, Sweeney P, Brady AP. Renal artery embolisation in the palliative treatment of renal carcinoma. Br J Radiol. 2007;80:96-102.

35. Marx FJ, Chaussy C, Moser E. [Limitations and hazards of palliative renal tumor embolization]. Urologe A.
1982;21:206-10.

36. Onishi T, Oishi Y, Yanada S, Abe K, Hasegawa T, Maeda S. Prognostic implications of histological features in patients with chromophobe cell renal carcinoma. BJU Int. 2002;90:529-32.

37. Ginat DT, Saad WEA, Turba UC.

Transcatheter renal artery embolization: clinical applications and techniques. Tech Vasc Interv Radiol. 2009;12:224-39. 